\title{
Bullous Fixed Drug Eruption Induced by Paracetamol: Report of a Pediatric Case
}

\author{
Massimiliano Nino Maria Grazia Francia Claudia Costa \\ Massimiliano Scalvenzi \\ Department of Dermatology, University Federico II of Naples, Naples, Italy
}

\section{Key Words}

Fixed drug eruptions · Paracetamol · Children

\begin{abstract}
Drug eruptions are among the most common adverse drug reactions, affecting approximately $3 \%$ of hospitalized patients. A fixed drug eruption (FDE) is a distinct druginduced reaction pattern that characteristically recurs at the same skin or mucosal site. We report a case of a 2-year-old girl with bullous FDE due to Tachipirina syrup, a preparation containing paracetamol, a commonly used nonsteroidal anti-inflammatory drug in Italy.
\end{abstract}

\section{Introduction}

Fixed drug eruption (FDE) is a cutaneous adverse drug reaction commonly seen in children and adolescents that is frequently misdiagnosed, leading to recurrent eruptions when the offending drug is readministered [1-2]. The diagnostic hallmark is its recurrence at previously affected sites. Although the term FDE was introduced by Brocq in 1894, the phenomenon had been initially described by Brouns 5 years earlier [3]. Bullous FDEs are rare and require differentiation from other pediatric blistering diseases.

\section{Case Report}

We describe a 2-year-old girl with bullous FDE due to Tachipirina ${ }^{\circledR}$ syrup, a preparation containing paracetamol. The episode occurred in the course of a fever treated with paracetamol. $3 \mathrm{~h} \mathrm{after} \mathrm{drug}$ administration, the young patient developed a bullous lesion, $0.6 \mathrm{~cm}$ in diameter, located on the third finger of the right hand. In a further few hours the lesion extended to cover almost all the dorsal surface of the finger reaching $2.4 \mathrm{~cm}$ (fig. 1). She had not been subject to other drug intakes in the last month. Blood cell count, kidney and liver function, serum electrolytes, serum glucose, C-reactive protein, immunoglobulin levels were all within normal limits. The bacterial cultures were all negative. We proceeded to the emptying of the bullous lesion; then the young patient was treated with an oxide zinc 
paste for 3 weeks. Within 7 days the inflammation subsided and complete reepithelization was reached in 20 days. No scarring or milia formation ensued.

Two months later, patch testing was performed using SIDAPA (Società Italiana di Dermatologia Allergologica Professionale e Ambientale) standard series and paracetamol 5\% (F.I.R.M.A., Florence, Italy; Finn ${ }^{\circledR}$ chambers on Scanpor ${ }^{\circledR}$ tape, Epitest Ltd., Tuusula, Finland). Standard series were located on the back, while paracetamol 5\% was applied both on the back and on the site of past lesion (third finger of the right hand). The positive reactions (++) to paracetamol 5\% observed at day 2 and day 4 in the two sites confirmed the allergic origin of the case presented. There were no positive reactions to SIDAPA standard series. No controls were undertaken. Positive reactions spontaneously healed in one week on both sites.

\section{Discussion}

Paracetamol is a widely used analgesic-antipyretic with consistent safety profile and very low incidence of side effects. Its therapeutic indications are: fever, headache, myalgia and other painful events of various origin.

Toxic eruptions induced by paracetamol are rare and usually of a fixed type [4]. Bullous FDE is still more rare [5] and, when appearing in children, it requires differentiation from other pediatric blistering diseases, including bullous erythema multiforme, linear IgA bullous dermatosis, and bullous pemphigoid.

In the reported case we did not perform a skin biopsy for histological examination because the child's mother did not give her consent, likely because of the patient's very young age. Although up to date oral rechallenge is the most reliable method of identifying the causative drug, we thought it not ethical in this young patient.

Nevertheless, a positive patch test on the site of a past lesion is a standardized and safe diagnostic method. When it is not possible to perform a patch test on the site of FDE (for example on mucosal sites), a positive patch test on healthy skin can, however, represent a valid diagnostic tool in such cases. 
Fig. 1. $2.4 \mathrm{~cm}$ bullous lesion on the dorsal surface of the third finger of the right hand $(8 \mathrm{~h}$ after drug intake).

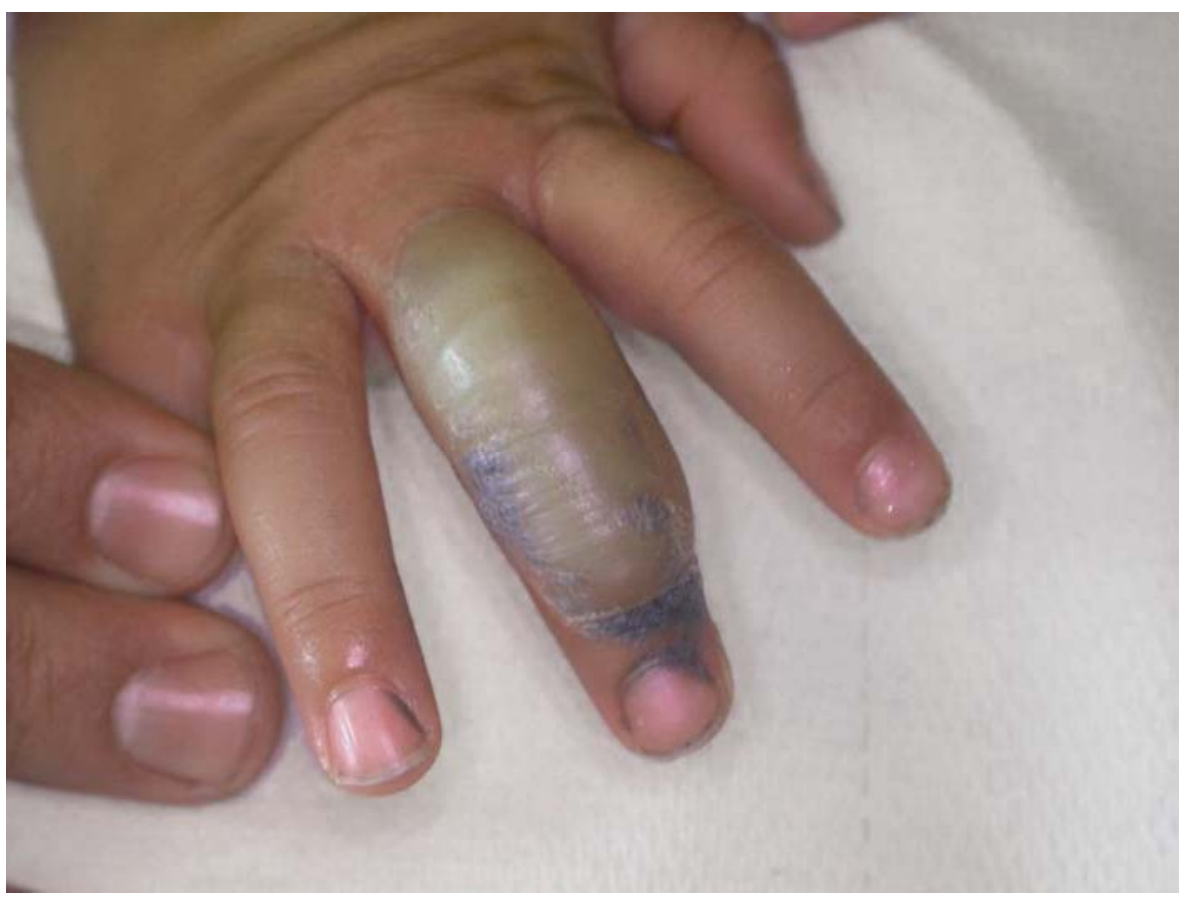




\section{References}

1 Shiohara T, Mizukawa Y: Fixed drug eruption: easily overlooked but needing new respect. Dermatology 2002;205:103-104.

2 Lee AY: Fixed drug eruptions. Incidence, recognition, and avoidance. Am J Clin Dermatol 2000;1:277-285.

-3 Silva A, Proenca E, Carvalho C, et al: Fixed drug eruption induced by paracetamol. Paediatr Dermatol 2001;18:163-164.

-4 Dogra S, Handa S: Bullous necrotizing fixed drug eruption in an infant. Pediatr Dermatol 2004;21:281-282.

5 Ayala F, Nino M, Ayala F, et al: Bullous fixed drug eruption induced by paracetamol: report of a case. Dermatitis 2006;17:160. 